\title{
Development of an assay for the detection and quantification of the measles virus nucleoprotein (N) gene using real-time reverse transcriptase PCR
}

\begin{abstract}
Correspondence
Hirokazu Kimura

kimhiro@nih.go.jp
\end{abstract}

Received 31 July 2008

Accepted 18 January 2009

\author{
Miho Akiyama, ${ }^{1} \dagger$ Hirokazu Kimura, ${ }^{1} \dagger$ Hiroyuki Tsukagoshi, ${ }^{2}$ \\ Katsuya Taira, ${ }^{3}$ Katsumi Mizuta, ${ }^{4}$ Mika Saitoh, ${ }^{2}$ Manami Nagano, ${ }^{5}$ \\ Asuka Sutoh, ${ }^{4}$ Masahiro Noda, ${ }^{6}$ Yukio Morita, ${ }^{2}$ Osamu Sakatsume, ${ }^{5}$ \\ Nobuhiko Okabe $^{1}$ and Masato Tashiro ${ }^{6}$
}

\author{
${ }^{1}$ Infectious Disease Surveillance Center, National Institute of Infectious Diseases, 4-7-1 Gakuen, \\ Musashimurayama, Tokyo 208-0011, Japan \\ ${ }^{2}$ Gunma Prefectural Institute of Public Health and Environmental Sciences, 378 Kamioki-machi, \\ Maebashi, Gunma 371-0052, Japan \\ ${ }^{3}$ Okinawa Prefectural Institute of Public Health and Environmental Sciences, 2085 Ozato, Nanjo, \\ Okinawa 910-1202, Japan \\ ${ }^{4}$ Yamagata Prefectural Institute of Public Health, 1-6-6 Tokamachi, Yamagata 990-0031, Japan \\ ${ }^{5}$ Molecular and Cellular Biology Division, Applied Biosystems Japan Ltd, 4-5-4 Hatchobori, \\ Chuo-ku, Tokyo 104-0032, Japan \\ ${ }^{6}$ Department of Virology III, National Institute of Infectious Diseases, 4-7-1 Gakuen, \\ Musashimurayama, Tokyo 208-0011, Japan
}

\begin{abstract}
We developed a new quantification method for the measles virus (MeV) nucleoprotein $(\mathrm{N})$ gene using real-time reverse transcriptase PCR. This method allowed us to quantify $10^{1}-10^{7}$ copies per reaction (corresponding to $5 \times 10^{-1}-5 \times 10^{5}$ copies $\mu^{-1}$ ) of the $\mathrm{MeV} \mathrm{N}$ gene. We also quantified the MeV N gene from the throat swabs of 22 patients with measles as well as the MeV genotypes A, D3, D5, D9 and H1 in viral suspensions derived from MeV-infected cells. As a result, $3.9 \times 10^{3}-5.2 \times 10^{6}$ copies $\mathrm{ml}^{-1}$ and $7.4 \times 10^{7}-2.0 \times 10^{8}$ copies $\mathrm{ml}^{-1}$ of the MeV genomes (N gene) were detected in the throat swabs and viral suspensions, respectively. No other viruses (enteroviruses, respiratory syncytial virus, human metapneumovirus or mumps virus) were detected in the assay. The results suggest that this method is applicable to the detection and quantification of some genotypes of $\mathrm{MeV}$.
\end{abstract}

\section{INTRODUCTION}

The measles virus $(\mathrm{MeV})$, genus Morbillivirus, family Paramyxoviridae, causes acute and highly contagious measles infection in humans (Griffin, 2007). The case fatality rate in developing countries is generally in the range of $1-5 \%$, but may be as high as $25 \%$ in populations with high levels of malnutrition and poor access to health care (http://www.who.int/mediacentre/factsheets/fs286/en/print. html). Therefore, the World Health Organization has focused on the infection as an eliminative disease (WHO \& United Nations Children's Fund, 2001; WHO, 2006).

†These authors contributed equally to this work.

Abbreviations: $C_{t}$, threshold cycle; RT-PCR, reverse transcriptase PCR.

The GenBank/EMBL/DDBJ accession numbers for the measles virus nucleoprotein gene sequences are AB447494-AB447515.
A genetic detection method for $\mathrm{MeV}$, i.e. reverse transcriptase nested PCR (nested RT-PCR), is widely used (Morita et al., 2007). While this method may be very sensitive, it can take more than $8 \mathrm{~h}$ to detect $\mathrm{MeV}$. Moreover, cross-contamination during the RT-PCR processes may cause significant problems (Llop et al., 2000; Drago et al., 2004). To improve these problems, recently a real-time reverse transcriptase PCR (real-time RT-PCR) method using specific primers and probes has been applied for the detection of various virus genomes (Mackay et al., 2002). This method is highly sensitive and specific, and quantitative.

In this study, we developed a sensitive and quantitative assay for the detection of the $\mathrm{MeV}$ nucleoprotein $(\mathrm{N})$ gene in clinical specimens (throat swabs) and viral suspensions derived from $\mathrm{MeV}$-infected cells (Vero/SLAM cells). 


\section{METHODS}

Clinical specimens. Throat swabs were obtained from 22 patients with measles. The samples were centrifuged at $3000 \mathrm{~g}$ for $30 \mathrm{~min}$ at $4{ }^{\circ} \mathrm{C}$ and the supernatants were used in this study (Morita et al., 2007). The specimens were stored at $-80{ }^{\circ} \mathrm{C}$ until used. Detailed data concerning patients, copies of the $\mathrm{MeV} \mathrm{N}$ gene in throat swabs and genotypes are shown in Table 1.

Viruses, virus propagation and RNA extraction. Five $\mathrm{MeV}$ strains (genotype A, CAM-70; genotype D3, MVi/Okinawa.JPN/31.01[D3]; D5, MVi/Okinawa.JPN/03.03[D5]; D9, MVi/Yamagata.JPN/3.04[D9]; and $\mathrm{H} 1, \mathrm{MVi} /$ Okinawa.JPN/14.03[H1]) and the 22 throat swab specimens were propagated in SLAM (signalling lymphocyte activation molecule, CD150)-expressing Vero cells (Vero/SLAM cells, kindly donated by Dr Y. Yanagi, Faculty of Medicine, Kyushu University) with Opti-MEM (Invitrogen). Vero and Vero/SLAM cells were maintained as previously described (Ono et al., 2001). Each virus suspension titre was $10^{6}-10^{7} \operatorname{TCID}_{50}(0.1 \mathrm{ml})^{-1}$ (tissue culture infective dose $50 \%$ ). MeV RNA was extracted from $200 \mu$ lof the throat swab specimen or the viral suspension using a High Pure Viral RNA kit (Roche Diagnostics). The extracted RNA was then suspended in $50 \mu \mathrm{l}$ DNase/RNase-free water with $1 \mathrm{U}$ RNase inhibitor (Applied Biosystems) $\mu \mathrm{l}^{-1}$.

Design of primers and probe. We aligned nucleotide sequences of the $\mathrm{N}$ gene from various genotypes of the reference $\mathrm{MeV}$ strains (genotype A, B1, B2, B3, C1, C2, D1-D10, E, F, G1, G2, G3, H1 and H2), as previously described (WHO, 2005; Morita et al., 2007). Based on these data, we designed new primers and a TaqMan probe using Primer Express (R) version 1.5 software (Applied Biosystems) (Thomas et al., 2007) (Table 2).
Preparation of control plasmid. To prepare the control plasmids, the $\mathrm{N}$ gene (position 1368-1616, $249 \mathrm{bp}$ ) in the following genotypes was amplified by PCR using the newly designed primers: genotype A, CAM-70 (GenBank accession no. U03650); genotype D3, MVi/ Okinawa.JPN/31.01[D3] (AB435245); D5, MVi/Okinawa.JPN/ 03.03[D5] (AB435246); D9, MVi/Yamagata.JPN/3.04[D9] (AB186905); and $\mathrm{H} 1, \mathrm{MVi} / \mathrm{Okinawa.JPN} / 14.03[\mathrm{H1}$ (AB435247). The products were cloned into a pCR2.1-TOPO vector (Invitrogen) and purified with a High Pure Plasmid Isolation kit (Roche Diagnostics), according to the manufacturer's instructions. The concentration of the plasmid was determined by measuring the $A_{260}$. The DNA sequence was confirmed by sequencing, using a Big Dye Terminator version 1.1 Cycle Sequencing kit (Applied Biosystems) (Morita et al., 2007).

Synthetic RNA of the N gene. Synthetic RNA of the MeV N gene (full-length, genotype A, position 108-1685, 1578 nucleotides) was prepared using the T7 MEGAscript kit (Applied Biosystems) as previously described (Hummel et al., 2006). Synthetic RNA was treated with DNase and purified. The size of the synthetic RNA was determined by ethidium bromide staining on denatured agarose gel. The concentration of the synthetic RNA of the $\mathrm{N}$ gene was determined by measuring the $A_{260}$.

Procedures of quantitative real-time RT-PCR. The reverse transcription mixture contained $10 \mu \mathrm{l}$ template RNA from the throat swab specimens, $4 \mu \mathrm{l}$ random hexamer $(20 \mathrm{pmol}), 4 \mu \mathrm{l} 5 \times$ RT-PCR buffer, $1 \mu$ l PrimeScript RT Enzyme Mix I (Takara) containing 10 units RNase inhibitor $\mu \mathrm{l}^{-1}$ (Applied Biosystems) and $1 \mu \mathrm{l}$ DNase- and RNase-free distilled water. The samples were incubated for $15 \mathrm{~min}$ at $37{ }^{\circ} \mathrm{C}$ then for $5 \mathrm{~s}$ at $85{ }^{\circ} \mathrm{C}$. PCR amplification was performed with a 7500 Fast Real-Time PCR System (Applied Biosystems) under the following conditions: initial uracil- $N$-glycosylase (UNG) amplicon

Table 1. Patient data, copies of the $\mathrm{N}$ gene of measles virus in throat swab, and genotype

\begin{tabular}{|c|c|c|c|c|c|c|c|}
\hline Patient & Strain & Onset of rash & Sampling date & Copies $^{\star}$ & Genotype & Vaccination & $\begin{array}{c}\text { GenBank } \\
\text { accession no. }\end{array}$ \\
\hline 1 & MVs/Okinawa.JPN/15.08/3 & 4 April 2008 & 4 April 2008 & $4.4 \times 10^{5}$ & D5 & Unknown & AB447511 \\
\hline 3 & MVs/Okinawa.JPN/16.08/2 & 18 April 2008 & 14 April $2008 \dagger$ & $6.8 \times 10^{3}$ & D5 & Unknown & AB447512 \\
\hline 4 & MVs/Okinawa.JPN/16.08/3 & 16 April 2008 & 17 April 2008 & $8.4 \times 10^{4}$ & D5 & Unknown & AB447514 \\
\hline 5 & MVs/Okinawa.JPN/18.08/1 & 29 April 2008 & 29 April 2008 & $1.6 \times 10^{5}$ & D5 & Yes & AB447515 \\
\hline 7 & MVs/Okinawa.JPN/37.06 & 11 September 2006 & 12 September 2006 & $8.9 \times 10^{4}$ & D5 & No & AB447495 \\
\hline 8 & MVs/Okinawa.JPN/38.06 & 19 September 2006 & 22 September 2006 & $5.3 \times 10^{5}$ & D5 & No & AB447494 \\
\hline 9 & MVs/Okinawa.JPN/39.06/1 & 27 September 2006 & 27 September 2006 & $5.2 \times 10^{6}$ & D5 & Unknown & AB447496 \\
\hline 10 & MVs/Okinawa.JPN/39.06/2 & 27 September 2006 & 27 September 2006 & $1.2 \times 10^{5}$ & D5 & Yes & AB447497 \\
\hline 11 & MVs/Okinawa.JPN/39.06/3 & 28 September 2006 & 28 September 2006 & $3.4 \times 10^{6}$ & D5 & No & AB447498 \\
\hline 12 & MVs/Okinawa.JPN/39.06/5 & 28 September 2006 & 28 September 2006 & $2.6 \times 10^{5}$ & D5 & No & AB447499 \\
\hline 17 & MVs/Okinawa.JPN/42.06/1 & 21 October 2006 & 20 October $2006 \dagger$ & $6.0 \times 10^{4}$ & D5 & No & AB447504 \\
\hline 18 & MVs/Okinawa.JPN/45.06/1 & 7 November 2006 & 7 November 2006 & $2.0 \times 10^{4}$ & D5 & Unknown & AB447505 \\
\hline 19 & MVs/Okinawa.JPN/45.06/2 & 6 November 2006 & 7 November 2006 & $1.3 \times 10^{5}$ & D5 & Unknown & AB447506 \\
\hline 20 & MVs/Okinawa.JPN/45.06/3 & 7 November, 2006 & 7 November 2006 & $3.9 \times 10^{3}$ & D5 & Unknown & AB447507 \\
\hline 21 & MVs/Okinawa.JPN/48.06 & 30 November 2006 & 2 December 2006 & $1.1 \times 10^{5}$ & D5 & No & AB447508 \\
\hline 22 & MVs/Okinawa.JPN/50.06 & 14 December 2006 & 15 December 2006 & $1.6 \times 10^{5}$ & D5 & Yes & AB447509 \\
\hline
\end{tabular}

${ }^{\star}$ Copies are expressed as $\mathrm{ml}^{-1}$ of throat swab.

$\dagger$ Sampling was performed the day that fever appeared. 
Table 2. Primer and probe sequences used for MeV real-time RT-PCR

\begin{tabular}{|llcc|}
\hline Primer or probe & \multicolumn{1}{c|}{ Sequence $\left(\mathbf{5}^{\prime} \rightarrow \mathbf{3}^{\prime}\right)^{*}$} & Polarity $\boldsymbol{y}^{*}$ & Location $\ddagger$ \\
\hline Primers & & & \\
MV-F & CAS RGT GAT CAA ART GRR ARY GAG CT & + & $1368-1393$ \\
MV-R & YCC TGC CAT GGY YTG CA & - & $1600-1616$ \\
Probe $\$$ & & - & $1530-1546$ \\
MV-T & FAM-TCY GAT RCA GTR TCA AT-MGB-NQF & - & \\
\hline
\end{tabular}

${ }^{*}$ Mix bases in degenerate primers and probes are as follows: $S=G / C, R=A / G, Y=C / T$.

$\dagger+$, Sense; - , antisense.

$\ddagger$ Corresponding nucleotide position of the MVi/Edmonston-wt.USA/54[A] strain full-length genome (GenBank accession no. AF266288).

§Probe is labelled with 6-carboxyfluorescein (FAM) reporter dye at the $5^{\prime}$-end, and with minor groove binder (MGB)non-fluorescent quencher (NQF) at the 3 '-end of the oligonucleotide.

degradation $\left(50{ }^{\circ} \mathrm{C}\right.$ for $\left.2 \mathrm{~min}\right)$ and denaturation of UNG at $95{ }^{\circ} \mathrm{C}$ for 10 min to activate DNA polymerase, then 50 cycles of amplification with denaturation at $95{ }^{\circ} \mathrm{C}$ for $15 \mathrm{~s}$, and annealing and extension at $58{ }^{\circ} \mathrm{C}$ for $1 \mathrm{~min}$. Amplification data were collected and analysed with Sequence Detector software version 1.3 (Applied Biosystems). A 10fold serial dilution of standard cDNA plasmids $\left(10^{7}-10^{1}\right.$ copies of genotype A, D3, D5, D9 or H1) was used for the quantification of copies as a standard curve assay.

Genotyping of MeV. We performed genotyping of $\mathrm{MeV}$ isolated from the clinical samples. $\mathrm{N}$ gene amplification and nucleotide sequencing were performed as previously described (Takeda et al., 1999; Morita et al., 2007). Nucleotide sequences of the partial $\mathrm{N}$ gene of the $\mathrm{MeV}$ were analysed phylogenetically using the CLUSTAL W program available on the DNA Data Bank of Japan (DDBJ) homepage (http://clustalw.ddbj.nig.ac.jp/top-e.html) and TreeExplorer (Version 2.12) (http://evolgen.biol.metro-u.ac.jp/TE/). Evolutionary distances were estimated using Kimura's two-parameter method and phylogenic trees were constructed using the neighbour-joining method (Saitou \& Nei, 1987). The reliability of the tree was estimated using 1000 bootstrap replications.
Specificity assay. To examine the specificity of the real-time RTPCR assay, eight enterovirus samples (echovirus types 9 and 18; coxsackie viruses A16, B2 and B5; enterovirus 71; and poliovirus types 1 and 3 ) and 18 respiratory virus samples (five strains of respiratory syncytial virus, five of mumps virus, four of human metapneumovirus, two of influenza subtype A and two of influenza subtype B) were tested. The titres of these viruses ranged from $10^{3}$ to $10^{5} \mathrm{TCID}_{50}$ $(0.1 \mathrm{ml})^{-1}$.

\section{RESULTS AND DISCUSSION}

\section{Optimization of primer and probe concentrations in the present assay}

It is suggested that primer and probe concentrations affect sensitivity and specificity of real-time RT-PCR (Hashimoto et al., 2007). Thus we optimized the primer and probe concentrations as previously described (Hummel et al.,

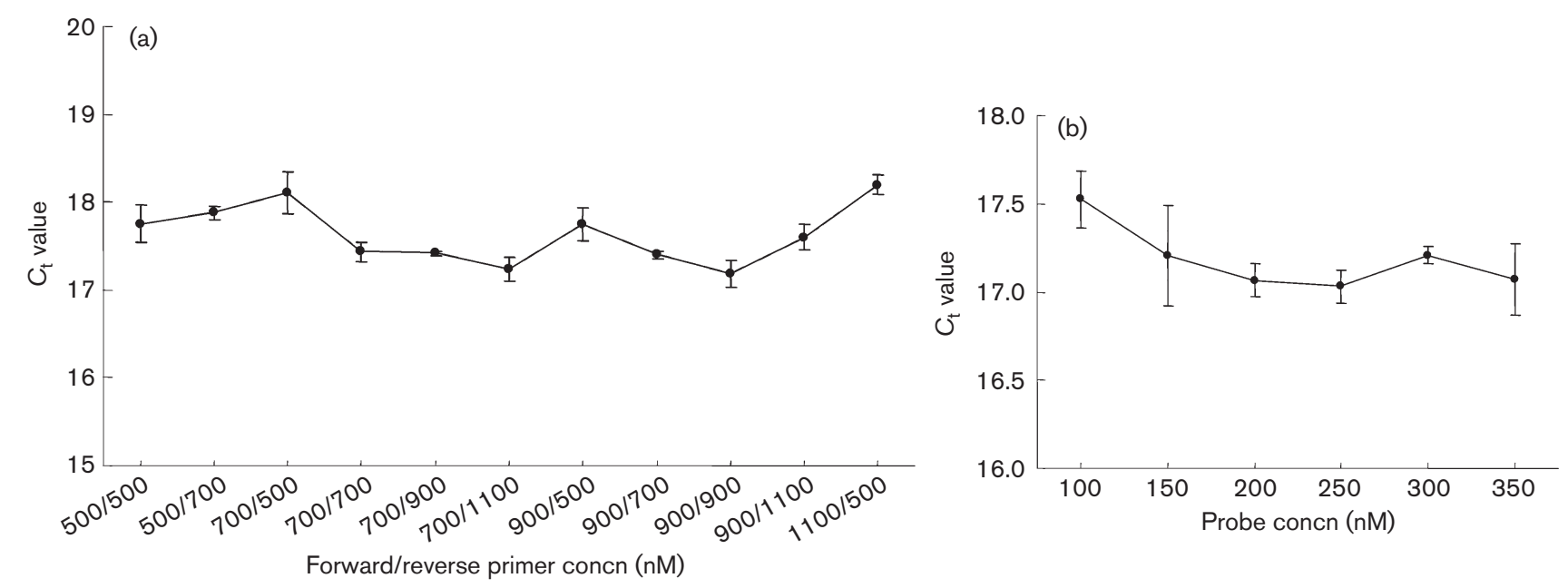

Fig. 1. Optimization of primer and probe concentrations in the present assay. Here, $10^{7}$ copies of synthetic RNA of the $\mathrm{N}$ gene (full-length, genotype A) were used. The $x$-axis indicates the concentrations of primer or probe and the $y$-axis indicates the $C_{t}$ value. Results are expressed as mean \pm SD from three independent experiments. Detailed procedures are described in the text. 
2006). The $C_{\mathrm{t}}$ (threshold cycle) values derived from the amplification plots for the present primer (ranging from 500 to $1100 \mathrm{nM}$ ) and probe (ranging from 100 to $350 \mathrm{nM}$ ) are shown in Fig. 1(a) and Fig. 1(b), respectively. In the present assay, $900 \mathrm{nM}$ of both forward and reverse primers and $250 \mathrm{nM}$ of probe showed the lowest $C_{\mathrm{t}}$ values. From these data, quantitative real-time PCR was carried out in a $20 \mu \mathrm{l}$ reaction volume using a TaqMan Universal PCR Master Mix (Applied Biosystems) containing $2 \mu \mathrm{l}$ cDNA, $900 \mathrm{nM}$ each primer (MV-F and MV-R) and $250 \mathrm{nM}$ TaqMan MGB probe (MV-T).

\section{Linearity, sensitivity and specificity of the real- time RT-PCR}

To assess the linearity of the quantitative real-time RTPCR, we prepared 10 -fold serial dilutions $\left(10^{7}-10^{1}\right.$ copies $)$ of cDNA plasmids (genotype A, D3, D5, D9 or H1), measured using newly designed primers and a probe. A representative standard curve and amplification plots using the standard plasmid (genotype A) are shown in Fig. 2(a, b). Good linearity was obtained from $10^{1}$ to $10^{7}$ copies per reaction $\left(R^{2}=0.9996\right)$ using other genotype-cloned plasmids (genotype D3, D5, D9 or H1) (data not shown). Next, to address the issue of sensitivity of the present assay, we prepared 10 -fold serial dilutions $\left(10^{7}-10^{1}\right.$ copies $)$ of synthetic RNA of the $\mathrm{MeV} \mathrm{N}$ gene (genotype A) and measured copy numbers as previously described (Fig. 3a) (Hummel et al., 2006). As a result, a good coefficient factor $\left(R^{2}=0.9987\right)$ was obtained from $10^{1}$ to $10^{7}$ copies per reaction (corresponding to $5 \times 10^{-1}-5 \times 10^{5}$ copies $\mu \mathrm{l}^{-1}$ ). The results suggest that the reliable measurement range of the present assay is $10^{1}-10^{7}$ copies per reaction. In addition, we prepared viral RNA (corresponding to $10^{1}-$ $10^{5}$ copies per reaction) of some $\mathrm{MeV}$ genotypes (genotype A, D3, D5, D9 or H1) using a dilution series of the viral suspensions and quantified copy numbers of their $\mathrm{N}$ gene. Good linearities (from $10^{1}$ to $10^{5}$ copies per reaction) as well as synthetic RNA were obtained for all genotypes (Fig. 3b). No other viruses such as enteroviruses, respiratory syncytial viruses, mumps viruses and influenza viruses (subtypes A and B) were detected with the present assay. The literature does contain some reports regarding the detection and quantification of $\mathrm{MeV}$ using sensitive real-time PCR methods (Hummel et al., 2006; Thomas et al., 2007). For example, Thomas et al. (2007) developed a quantification assay for the haemagglutinin $(\mathrm{H})$ gene in $\mathrm{MeV}$ using a real-time PCR method and Hummel et al. (2006) developed a real-time RT-PCR method targeting multiple genes [nucleoprotein $(\mathrm{N})$, fusion $(\mathrm{F})$ and $\mathrm{H}$ genes]. In these methods, the sensitivity limits were two and ten copies per reaction, respectively (Hummel et al., 2006; Thomas et al., 2007). Thus these values were very similar to those of our method, even though the target gene and/or region of $\mathrm{MeV}$ were different. Taken together, the results suggest that our method is sensitive, specific and quantitative for the $\mathrm{MeV} \mathrm{N}$ gene, and is applicable to the quantification of various $\mathrm{MeV}$ genotypes.

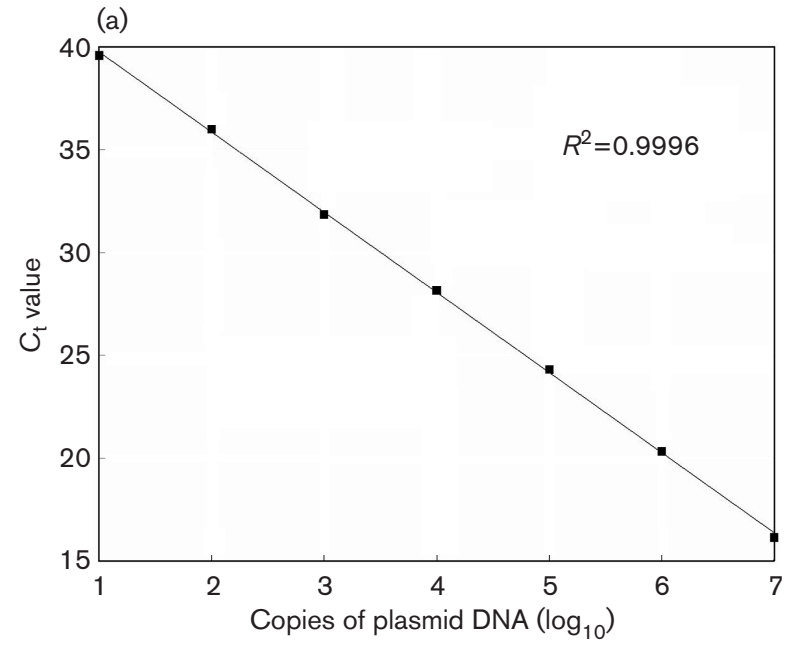

(b)

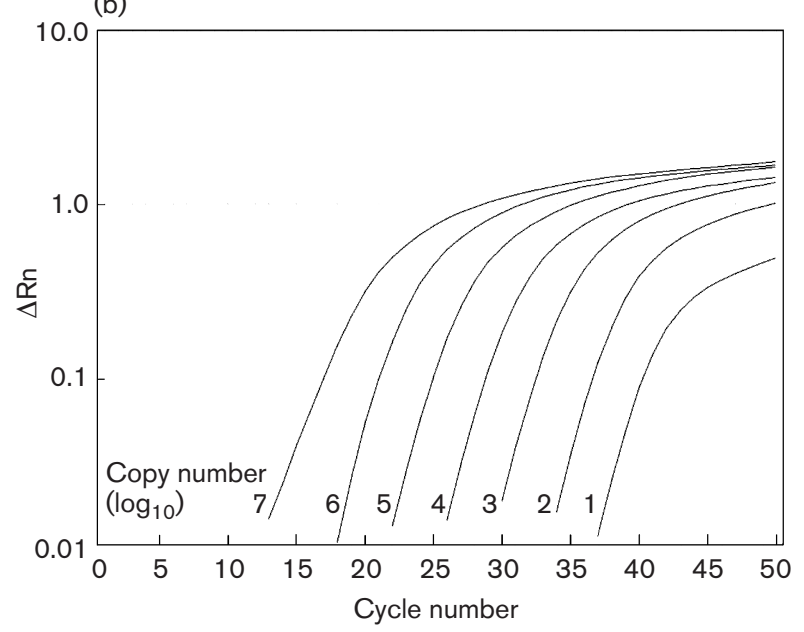

Fig. 2. Standard curve and amplification plots of quantification of the nucleoprotein $(\mathrm{N})$ gene of MeV. A 10-fold serial dilution (10 ${ }^{7}-$ $10^{1}$ copies per reaction) of cDNA plasmid (genotype A) was used. (a) The $x$-axis indicates the logarithmic concentration $\left(\log _{10}\right)$ of the cDNA plasmid and the $y$-axis indicates the $C_{t}$ value. (b) The $x$-axis indicates the $C_{t}$ value and the $y$-axis indicates $\Delta \mathrm{Rn}$ (adjusted fluorescence intensity). Detailed procedures are described in the text.

\section{Isolation, genotype and quantification of MeV from throat swabs of patients with measles}

$\mathrm{MeV}$ was isolated from all specimens and the genotype was confirmed as D5 by phylogenetic analysis (Table 1). In addition, throat swabs from patients with measles contained $3.9 \times 10^{3}-5.2 \times 10^{6}$ copies $\mathrm{ml}^{-1}$ of the $\mathrm{N}$ gene. No significant differences were found between intervals of onset of rash and sampling date and copies of $\mathrm{MeV}$ in the throat swab specimens. In these patients, the lowest number of copies of $\mathrm{MeV}$ from raw data was 15.4 copies per reaction (corresponding to $3.9 \times 10^{3}$ copies $\mathrm{ml}^{-1}$ in throat swab, multiplication factor 250), suggesting that this value represents a reliable zone of the standard curve in the 

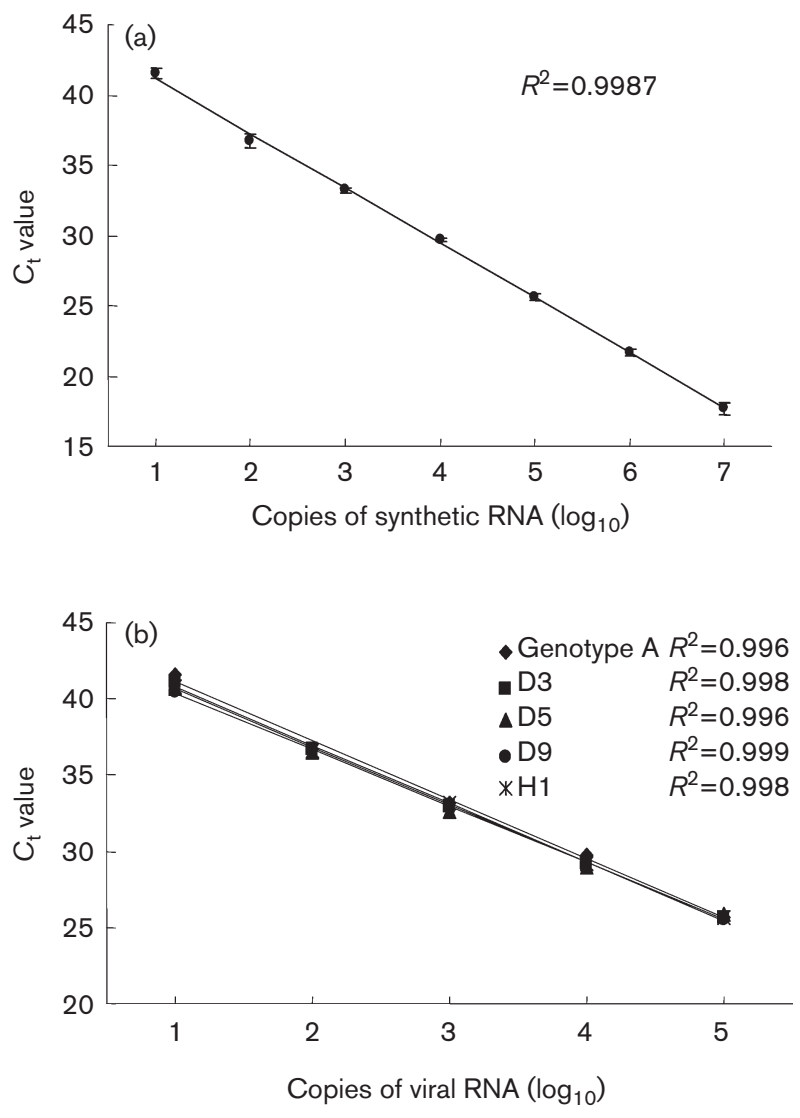

Fig. 3. Linearity of the real-time RT-PCR assay. (a) A 10-fold serial dilution $\left(10^{7}-10^{1}\right.$ copies per reaction) of synthetic RNA of the MeV N gene (full-length, genotype A) was used. (b) A 10-fold serial dilution $\left(10^{5}-10^{1}\right.$ copies per reaction) of viral RNA of each genotype (genotype A, D3, D5, D9 or H1) was used. In Fig. 3(a, b), the $x$-axis indicates the logarithmic concentration $\left(\log _{10}\right)$ of the synthetic or viral RNA and the $y$-axis indicates the $C_{t}$ value. Results are expressed as mean \pm SD from three independent experiments. Detailed procedures are described in the text.

present assay. In this study, we did not measure other $\mathrm{MeV}$ genotypes (D3, D9 or H1) in the throat swab specimens since we were unable to obtain them from patients with measles. Thus we quantified the A, D3, D5, D9 and H1 genotypes of the $\mathrm{MeV} \mathrm{N}$ gene in viral suspensions derived from $\mathrm{MeV}$-infected Vero/SLAM cells and some plasmid controls. As a result, each viral suspension contained $7.4 \times 10^{7}-2.0 \times 10^{8}$ copies $\mathrm{ml}^{-1}$. In Japan, over the past 10 years, domestic measles outbreaks have occurred in 1998, 2001, 2006 and 2007 (Kubo et al., 2002; Zhou et al., 2003; Nakajima et al., 2003; Morita et al., 2007). The predominant $\mathrm{MeV}$ genotype detected during these outbreaks was D5, although a small number of other genotypes including D3, D9 and $\mathrm{H} 1$ were also detected. Thus it may be important to confirm detection of these $\mathrm{MeV}$ genotypes in Japan. In the present study, in order to detect and quantify $\mathrm{MeV}$ genotypes and to prevent cross-reactions of the $\mathrm{N}$ gene in other paramyxoviruses such as mumps virus, respiratory syncytial virus and parainfluenza viruses, we targeted a specific region of the $\mathrm{MeV} \mathrm{N}$ gene. However, the sequences of this region (the PCR target region) may be variable (Griffin, 2007). As such, it may be necessary to modify the primers/probe sequences in the future when mismatches are found to occur in them. Furthermore, we did not examine one-step real-time RT-PCR in this study, a method which may be advantageous when large numbers of samples are screened (Hummel et al., 2006). Thus in future work we will consider the need to introduce this technique in the present assay. The present results indicate that the newly developed assay may be applicable for the detection and quantification of $\mathrm{MeV}$ from clinical specimens (throat swabs) and viral suspensions.

\section{ACKNOWLEDGEMENTS}

We thank Dr H. Yokoi and Ms T. Kitahashi for the helpful discussions. This work was partly supported by Research on Emerging and Re-emerging Infectious Diseases, Labour, and Welfare Programs from the Ministry of Health, Labour, and Welfare, Japan.

\section{REFERENCES}

Drago, L., Lombardi, A., De Vecchi, E., Giuliani, G., Bartolone, R. \& Gismondo, M. R. (2004). Comparison of nested PCR and real time PCR of Herpesvirus infections of central nervous system in HIV patients. BMC Infect Dis 4, 55.

Griffin, D. E. (2007). Measles virus. In Fields Virology, 5th edn, pp. 1551-1585. Edited by D. M. Knipe, P. M. Howley, D. E. Griffin, R. A. Lamb, M. A. Martin, B. Roizman \& S. E. Straus. Philadelphia: Lippincott Williams \& Wilkins.

Hashimoto, Y., Valles, S. M. \& Strong, C. A. (2007). Detection and quantitation of Solenopsis invicta virus in fire ants by real-time PCR. J Virol Methods 140, 132-139.

Hummel, K. B., Lowe, L., Bellini, W. J. \& Rota, P. A. (2006). Development of quantitative gene-specific real-time RT-PCR assays for the detection of measles virus in clinical specimens. J Virol Methods 132, 166-173.

Kubo, H., Iritani, N., Murakami, T. \& Haruki, K. (2002). Isolation of a wild type measles virus classified as genotype $\mathrm{H} 1$ in Osaka city. Jpn J Infect Dis 55, 177-179.

Llop, P., Bonaterra, A., Peñalver, J. \& López, M. M. (2000). Development of a highly sensitive nested-PCR procedure using a single closed tube for detection of Erwinia amylovora in asymptomatic plant material. Appl Environ Microbiol 66, 2071-2078.

Mackay, I. M., Arden, K. E. \& Nitsche, A. (2002). Real-time PCR in virology. Nucleic Acids Res 30, 1292-1305.

Morita, Y., Suzuki, T., Shiono, M., Shiobara, M., Saitoh, M., Tsukagoshi, H., Yoshizumi, M., Ishioka, T., Kato, M. \& other authors (2007). Sequence and phylogenetic analysis of the nucleoprotein $(\mathrm{N})$ gene in measles viruses prevalent in Gunma, Japan, in 2007. Jpn J Infect Dis 60, 402-404.

Nakajima, N., Matsuda, T., Ono, T., Murakami, H., Tokutake, T., Matsumiya, C., Tateyama, S., Honjo, A., Katsuta, T. \& other authors (2003). Measles outbreak in a suburb of Tokyo, Japan, in 1998-1999. Scand J Infect Dis 35, 495-497. 
Ono, N., Tatsuo, H., Hidaka, Y., Aoki, T., Minagawa, H. \& Yanagi, Y. (2001). Measles viruses on throat swabs from measles patients use signaling lymphocytic activation molecule (CDw150) but not CD46 as a cellular receptor. J Virol 75, 4399-4401.

Saitou, N. \& Nei, M. (1987). The neighbor-joining method: a new method for reconstructing phylogenetic trees. Mol Biol Evol 4, 406425.

Takeda, M., Sakaguchi, T., Li, Y., Kobune, F., Kato, A. \& Nagai, Y. (1999). The genome nucleotide sequence of a contemporary wild strain of measles virus and its comparison with the classical Edmonston strain genome. Virology 256, 340-350.

Thomas, B., Beard, S., Jin, L., Brown, K. E. \& Brown, D. W. (2007).

Development and evaluation of a real-time PCR assay for rapid identification and semi-quantitation of measles virus. J Med Virol 79, 1587-1592.

WHO (2005). New genotype of measles virus and update on global distribution of measles genotypes. Wkly Epidemiol Rec 80, 347-351.

WHO (2006). Progress in reducing global measles deaths: 1999-2004. Wkly Epidemiol Rec 81, 90-94.

WHO \& United Nations Children's Fund (2001). Measles Mortality Reduction and Regional Elimination Strategic Plan 2001-2005. www.who.int/vaccines-documents/ and www.unicef.org

Zhou, J., Fujino, M., Inou, Y., Kumada, A., Aoki, Y., Iwata, S. \& Nakayama, T. (2003). H1 genotype of measles virus was detected in outbreaks in Japan after 2000. J Med Virol 70, 642-648. 